\title{
Extranodal marginal zone B-cell lymphoma involving superior rectus muscle: A clinicopathological case report
}

\author{
JUNICHI FUKUHARA, SATORU KASE, MIKA NODA, KAN ISHIJIMA and SUSUMU ISHIDA \\ Department of Ophthalmology, Hokkaido University Graduate School of Medicine, Kita-ku, Sapporo 060-8638, Japan
}

Received December 27, 2010; Accepted March 7, 2011

DOI: $10.3892 / \mathrm{ol} .2011 .272$

\begin{abstract}
A 66-year-old female had suffered from proptosis in the left eye (OS) and double vision for 1 month due to abnormality of the superior rectus muscle. Visual acuity was noted as 20/20 in both eyes (OU). Eye movement showed limited OS supraduction. Magnetic resonance imaging revealed an indistinct mass in the orbit involving the superior rectus muscle. A biopsy specimen of the orbital tumor led to the histological diagnosis of extranodal marginal zone B-cell lymphoma. Radiotherapy with a total dosage of 30 Gy was administered, which subsequently resolved the tumor. However, the supraduction limitation of ocular movement remained unchanged. Supraduction limitation is due to muscular contraction disorder of the superior rectus muscle, caused by direct lymphoma cell invasion.
\end{abstract}

\section{Case report}

Extranodal marginal zone B-cell lymphoma (EMZL) of mucosa-associated lymphoid tissue lymphoma arises from the marginal zone of mucosa-associated lymphoid tissue. EMZL is common among ocular adnexal malignant tumors; however, orbital lymphomas involving striated muscles are rare. This report examines an unusual case of orbital EMZL involving the superior rectus muscle.

A 66-year-old female suffering from proptosis of the left eye (OS) and double vision for 1 month was referred to our hospital due to an abnormality of the superior rectus muscle OS. Visual acuity was found to be $20 / 20$ in both eyes with a normal intraocular pressure. Extraocular examination demonstrated upper eyelid swelling without ocular pain. The fundus was normal. Hess screen analysis revealed supraduction OS (Fig. 1). The laboratory values, including a blood cell count, biochemistry, and thyroid hormones, were found to be normal.

Correspondence to: Dr Satoru Kase, Department of Ophthalmology, Hokkaido University Graduate School of Medicine, Nishi 7, Kita 15, Kita-ku, Sapporo 060-8638, Japan

E-mail: kaseron@med.hokudai.ac.jp

Key words: extranodal marginal zone B-cell lymphoma, superior rectus muscle, histopathology
No systemic abnormality was detected with the exception of the orbit. Initial magnetic resonance imaging (MRI) of the orbit revealed an indistinct mass in the superior orbit close to, or within, the superior rectus muscle (Fig. 2). Differential diagnoses of the mass lesion in the extraocular muscle were orbital tumor, Graves' disease, and orbital myositis. Biopsy of the orbital mass was performed.

A histological examination showed diffuse atypical lymphoid cell infiltration, mixed with plasma cells with Russell bodies. Lymphoid cells were present within fragmented striated muscles (Fig. 3A). Immunohistochemically, the atypical lymphoid cells were positive for CD20 and CD79a, markers for B-cells (Fig. 3B and C), and negative for CD3 and CD5, markers for T-cells. The immunohistochemical examination of immunoglobulin showed deviation to $\kappa$ chains in the infiltrating lymphoid cells (Fig. 3D).

The orbital tumor was diagnosed as EMZL involving the superior rectus muscle. Radiotherapy with a total dosage of $30 \mathrm{~Gy}$ was administered. The radiotherapy reduced the volume of the tumor (Fig. 4, red arrow), and the superior rectus muscle was clearly identified (Fig. 4, white arrow). The supraduction limitation in OS movement remained unchanged, although tumor recurrence was not observed one and a half years after radiotherapy.

\section{Discussion}

Since the border between the tumor and superior rectus muscle was not differentiated in the initial MRI of the orbit, the origin of the tumor remains to be determined. Histological examination showed atypical lymphoid cell infiltration within the fragmented striated muscle. Muscle fibers in the remaining striated muscle were clearly noted in the specimen, indicating that the muscle was not degenerative. Following radiotherapy, superior rectus muscle with a reduced tumor was visualized. Therefore, the clinicopathological findings indicate that EMZL did not arise in the extraocular muscle, but arose in the orbital soft tissue adjacent to the muscle, followed by invasion to the superior rectus muscle.

In this case, only superior muscle was involved in tumor cell invasion, presenting with supraduction limitation, but not inferior adduction limitation. These results suggest that supraduction limitation occurred due to muscular contraction disorder of the superior rectus and not due to restriction by an enlarged antagonist muscle as observed in Graves' disease. 


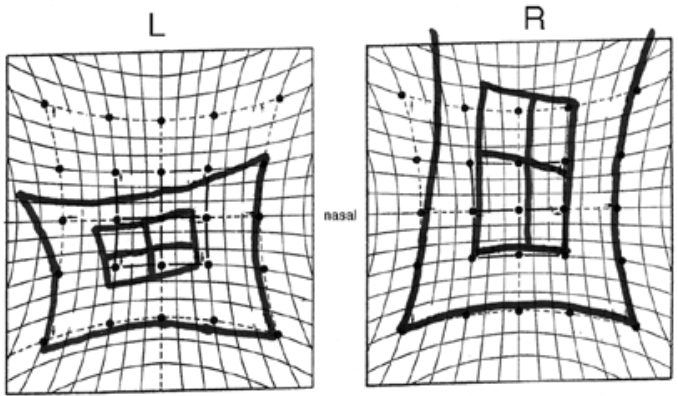

Figure 1. Hess screen analysis reveals difficulties with elevation of the left eye. L, left; R, right.

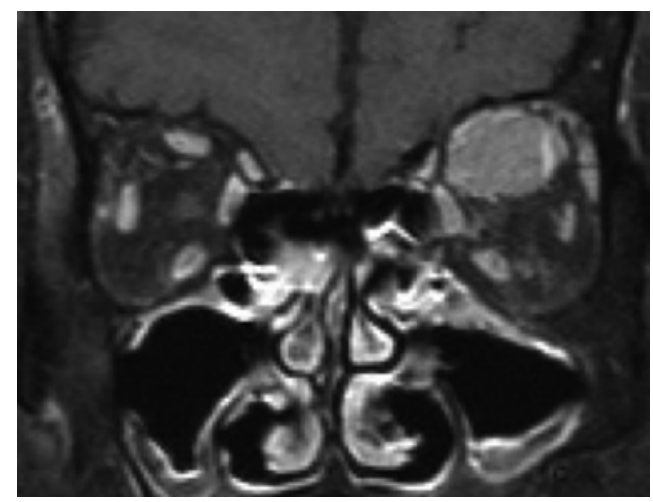

Figure 2. Magnetic resonance imaging shows a mass lesion at the level of the superior rectus muscle in the left orbit.

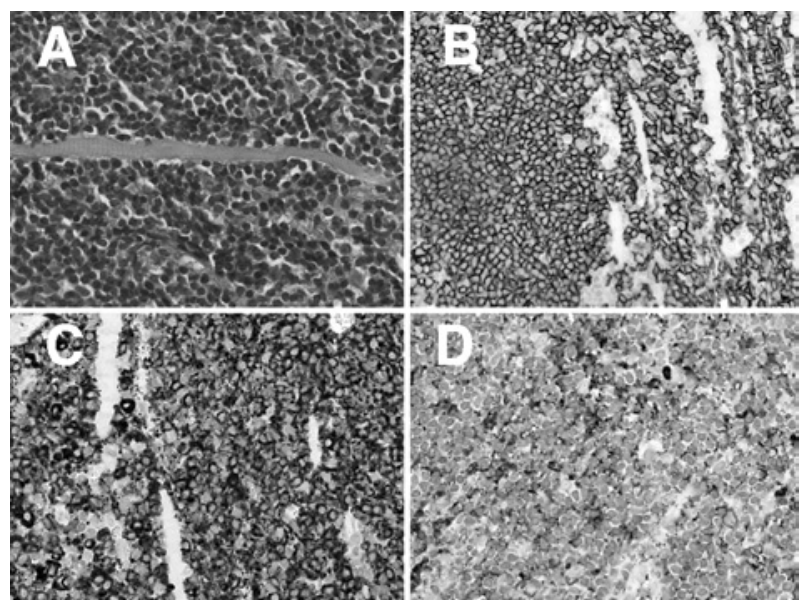

Figure 3. Histopathology of the biopsied orbital mass. (A) Typical lymphoid cells were diffusely infiltrated and mixed with fragmented striated muscle (hematoxylin-eosin). (B) Cytoplasmic immunoreacitivity for CD20, a marker for B-cells, is observed in tumor cells. (C) Cytoplasmic immunoreacitivity for CD79a, a marker for B-cells, is observed in tumor cells. (D) Immunohistochemical staining of immunoglobulin shows deviation to the $\kappa$ chain in the infiltrating lymphoid cells.

Together with the histological findings, it was noted that the extraocular muscle contraction disorder is caused by direct lymphoma cell invasion. Despite resolution of the tumor after irradiation, supraduction limitation was not improved, suggesting that irradiation led not only to tumor cell death, but also subsequent irreversible muscle fibrosis.

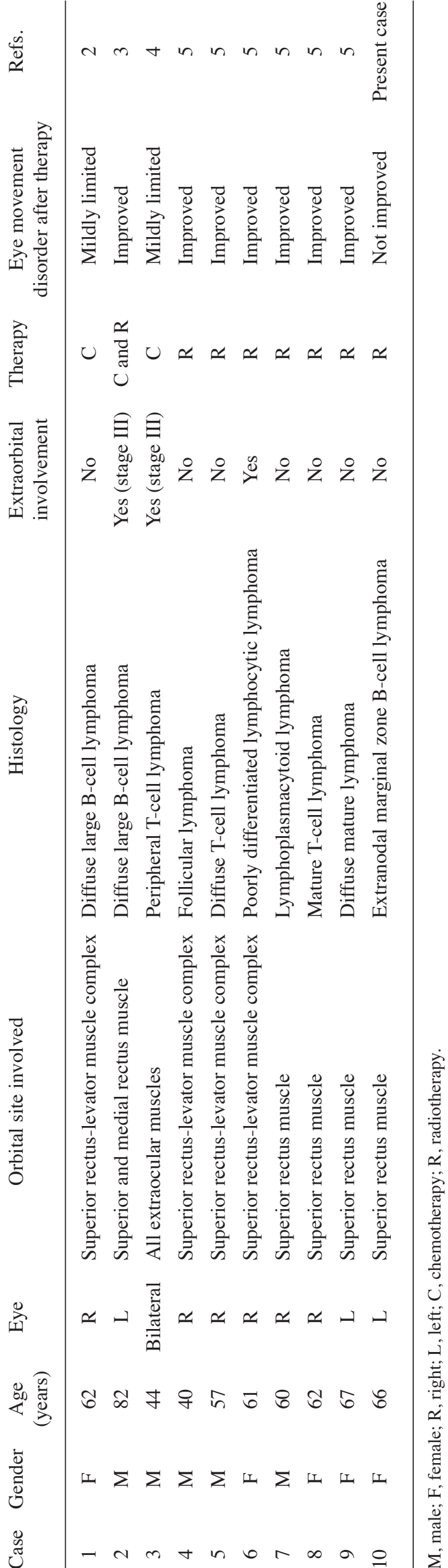



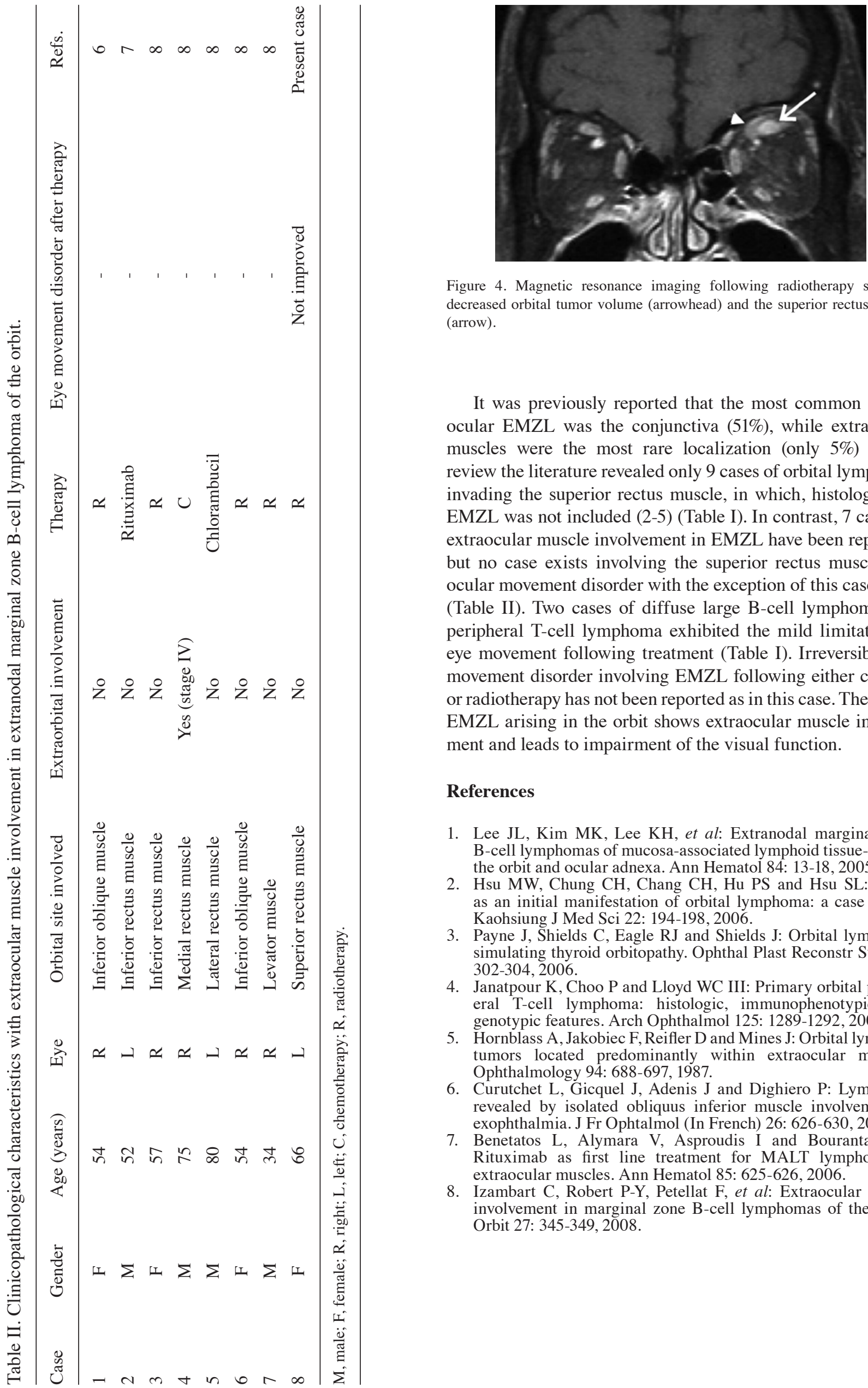

Figure 4. Magnetic resonance imaging following radiotherapy shows a decreased orbital tumor volume (arrowhead) and the superior rectus muscle (arrow).

It was previously reported that the most common site of ocular EMZL was the conjunctiva (51\%), while extraocular muscles were the most rare localization (only 5\%) (1). A review the literature revealed only 9 cases of orbital lymphoma invading the superior rectus muscle, in which, histologically, EMZL was not included (2-5) (Table I). In contrast, 7 cases of extraocular muscle involvement in EMZL have been reported, but no case exists involving the superior rectus muscle and ocular movement disorder with the exception of this case (6-8) (Table II). Two cases of diffuse large B-cell lymphoma and peripheral T-cell lymphoma exhibited the mild limitation of eye movement following treatment (Table I). Irreversible eye movement disorder involving EMZL following either chemoor radiotherapy has not been reported as in this case. Therefore, EMZL arising in the orbit shows extraocular muscle involvement and leads to impairment of the visual function.

\section{References}

1. Lee JL, Kim MK, Lee KH, et al: Extranodal marginal zone B-cell lymphomas of mucosa-associated lymphoid tissue-type of the orbit and ocular adnexa. Ann Hematol 84: 13-18, 2005.

2. Hsu MW, Chung CH, Chang CH, Hu PS and Hsu SL: Ptosis as an initial manifestation of orbital lymphoma: a case report. Kaohsiung J Med Sci 22: 194-198, 2006.

3. Payne J, Shields C, Eagle RJ and Shields J: Orbital lymphoma simulating thyroid orbitopathy. Ophthal Plast Reconstr Surg 22: 302-304, 2006.

4. Janatpour K, Choo P and Lloyd WC III: Primary orbital peripheral T-cell lymphoma: histologic, immunophenotypic, and genotypic features. Arch Ophthalmol 125: 1289-1292, 2007.

5. Hornblass A, Jakobiec F, Reifler D and Mines J: Orbital lymphoid tumors located predominantly within extraocular muscles. Ophthalmology 94: 688-697, 1987.

6. Curutchet L, Gicquel J, Adenis J and Dighiero P: Lymphoma revealed by isolated obliquus inferior muscle involvement in exophthalmia. J Fr Ophtalmol (In French) 26: 626-630, 2003.

7. Benetatos L, Alymara V, Asproudis I and Bourantas KL: Rituximab as first line treatment for MALT lymphoma of extraocular muscles. Ann Hematol 85: 625-626, 2006.

8. Izambart C, Robert P-Y, Petellat F, et al: Extraocular muscle involvement in marginal zone B-cell lymphomas of the Orbit. Orbit 27: 345-349, 2008 